\title{
Análise dos efeitos de comportamentos altruístas e egoístas em redes de transporte
}

\author{
Analysis of altruistic and egoistic effects in transportation networks \\ Análisis de los efectos del comportamiento altruista y egoísta en las redes de transporte
}

Recebido: 29/10/2021 | Revisado: 07/11/2021 | Aceito: 08/11/2021 | Publicado: 13/11/2021

\author{
Yuri Perez \\ ORCID: https://orcid.org/0000-0002-4463-6460 \\ Universidade Nove de Julho, Brasil \\ E-mail: yuriniet@gmail.com \\ Fabio Henrique Pereira \\ ORCID: https://orcid.org/0000-0002-6075-5566 \\ Universidade Nove de Julho, Brasil \\ E-mail: fabiohp@uni9.pro.br
}

\begin{abstract}
Resumo
Redes de transporte são infraestruturas fundamentais para a dinâmica de grandes centros urbanos. Essas estruturas estão sujeitas a congestionamentos, que trazem um forte impacto social, econômico e ambiental. Neste trabalho, foi construído um modelo de simulação baseado em agentes, a fim de investigar como comportamentos altruístas na seleção de rotas pode afetar os tempos de viagem e a distribuição de fluxo em uma rede de transporte. Métodos: A base de dados aberta OpenStreetMap foi utilizada para obter a estrutura da rede de transporte. A teoria de redes complexas foi usada para realizar a simulação e estimar os impactos do congestionamento. Simulando fluxos de mobilidade, foram analisados como os critérios de seleção de trajeto dos agentes influenciam nos níveis de congestionamento, nos comprimentos de trajeto e nos tempos de viagem. Resultados: altruísta reduz significativamente a propagação de congestionamentos e a formação de agrupamentos de vias congestionadas na rede de transporte, assim como reduz o tempo médio de viagem entre dois pontos, mas aumenta a distância média percorrida numa proporção menor.
\end{abstract}

Palavras-chave: Agentes; Rede de transporte; Altruísmo; Simulação; Congestionamento.

\begin{abstract}
Transport networks are fundamental infrastructure for the dynamics of large urban centers. These structures are subject to congestion, which have a strong social, economic and environmental impact. In this work, an agent-based simulation model was built in order to investigate how altruistic behavior in route selection can affect travel times and flow distribution in a transport network. Methods: OpenStreetMap open database was used to obtain the structure of the transport network. Complex network theory was used to perform the simulation and estimate the impacts of congestion. Simulating mobility flows, we analyzed how the agents' path selection criteria influence congestion levels, path lengths and travel times. Results: altruistic behavior significantly reduces the propagation of congestion and the formation of groupings of congested roads in the transport network, as well as reducing the average travel time between two points, but increasing the average distance traveled in a smaller proportion.
\end{abstract}

Keywords: Agents; Transportation network; Altruism; Simulation; Congestion.

\section{Resumen}

Las redes de transporte son una infraestructura fundamental para la dinámica de los grandes centros urbanos. Estas estructuras están sujetas a congestión, lo que tiene un fuerte impacto social, económico y ambiental. En este trabajo, se construyó un modelo de simulación basado en agentes para investigar cómo el comportamiento altruista en la selección de rutas puede afectar los tiempos de viaje y la distribución del flujo en una red de transporte. Métodos: Se utilizó la base de datos abierta OpenStreetMap para obtener la estructura de la red de transporte. Se utilizó la teoría de redes complejas para realizar la simulación y estimar los impactos de la congestión. Simulando flujos de movilidad, analizamos cómo los criterios de selección de ruta de los agentes influyen en los niveles de congestión, la longitud de las rutas y los tiempos de viaje. Resultados: comportamiento altruista reduce significativamente la propagación de la congestión y la formación de agrupaciones de carreteras congestionadas en la red de transporte, además de reducir el tiempo medio de viaje entre dos puntos, pero incrementando la distancia media recorrida en menor proporción.

Palabras clave: Agentes; Red de transporte; Altruismo; Simulación; Congestión. 


\section{Introdução}

Redes urbanas são sistemas complexos. Um sistema complexo é um sistema cujo comportamento global emerge das interações locais dos seus componentes (Ladyman et al., 2013). Em sistemas urbanos, estes comportamentos podem ser exemplificados pela formação de filas em trânsito, pelos fluxos de mobilidade entre centro e periferia e pela formação de microclimas urbanos.

Cidades são sistemas com duas dimensões de complexidade, uma representada pela geografia, recursos ambientais e infraestrutura física, e outra representada pelo comportamento humano (Portugali, 2016). Estas duas dimensões estão separadas por escalas temporais distintas, mas que se influenciam mutuamente. Sistemas complexos são, em geral, compostos por elementos que interagem entre si no tempo e no espaço, formando estruturas que se assemelham a redes. Estas redes podem ser representadas e estudadas por meio da teoria de redes complexas. A rede de transporte é um componente crítico no funcionamento de uma cidade. No Brasil, Moreira et al. (2019), após minuciosa investigação de boletins e documentos públicos, afirmam que demanda incontrolável e investimentos inconsistentes prejudicam a eficiência e a segurança dos sistemas de transporte no que tange a logística de cargas.

A rede de transporte pode ser considerada um sistema de infraestrutura crítica, cuja definição é dada pelo Decreto $\mathrm{N}^{\circ}$ 9.573, de 22 de novembro de 2018. Infraestrutura crítica é toda instalação, serviço ou sistema cuja interrupção ou destruição parcial ou total acarreta sério impacto ambiental, social, econômico ou político. Todo sistema de infraestrutura deve apresentar algum grau de resiliência e controlabilidade, a fim de ser gerenciável (Gao et al., 2016). As redes de transporte, especificamente, são aquelas que permitem o acesso a todos os outros sistemas de infraestrutura crítica, como a rede de atenção à saúde, por exemplo (Liu \& Song, 2020). A interação dos usuários cria uma força coletiva capaz de influenciar nas políticas de uso das redes de transporte. A interação entre agente e estrutura pode ser simulada com o auxílio de modelos multiagentes, que são compostos de unidades computacionais interagindo com o ambiente e uns com os outros por meio de regras préestabelecidas. Segundo Helbing e Balietti (2012) a modelagem baseada em agentes pode ser extremamente útil na simulação de sistemas complexos, e com o aumento da Macau e North (2005) definem modelos multiagentes como modelos compostos de unidades com comportamentos de diferentes níveis de complexidade, que podem interagir com o ambiente e uns com os outros por regras bem definidas.

Para a simulação de redes de transporte, é comum empregar modelos de alocação de tráfego. Nestes modelos, o principal objetivo é otimizar a alocação de tráfego nas arestas de forma a minimizar uma função. Para a alocação de tráfego baseada no equilíbrio de usuário, busca-se atingir um equilíbrio tal que nenhum usuário tenha incentivo para mudar de trajeto (Salman \& Alaswad, 2018). Mas também é possível modificar o modelo de alocação de tráfego tradicional para incorporar uma perspectiva altruísta na seleção de trajetos, tal que a função a ser otimizada minimze o tempo de viagem de todos os usuários (Eikenbroek et al.; 2021).

Os modelos baseados em agentes podem ser usados para a simulação de alocação de tráfego (Auld et al.; 2019). A vantagem dos modelos baseados em agentes é a possibilidade de estudar como a emergência de determinados cenários pode surgir de diferentes perfis de comportamento e como cada perfil de comportamento afeta na distribuição de tráfego. É possível, por exemplo, estudar como informação imperfeita acerca do tráfego afeta a alocação, assim como dar aos agentes a liberdade de reajustar o trajeto diante de mudanças observadas no sistema (Barthelemy \& Carletti, 2017). O uso de mecanismos de seleção de trajetos que busquem incoporar considerações acerca da sobrecarga dos tempos de viagem sobre o sistema já foi investigada a partir de diferentes abordagens, como por exemplo em Levy et al. (2018).

Em geral, agentes em sistemas de transporte são representados como indivíduos egoístas que buscam maximizar seu bem estar mesmo que isso prejudique outros agentes e torne o sistema como um todo menos eficiente. Sabe-se que a estrutura da rede onde os agentes se deslocam é um componente importante no incentivo de comportamentos altruístas de jogos em 
redes (Ben Akiva et al.; 2012). Levy et al. (2018) indica que ainda é preciso investigar a performance de comportamentos egoístas e altruístas em topologias de redes complexas.

Neste trabalho, busca-se simular o fluxo de agentes ao longo da região central da rede de transporte de São Paulo. Os agentes buscam selecionar trajetos entre dois pontos de forma a minimizar o custo do trajeto, e este custo é uma combinação do valor do tempo de trajeto (representando uma escolha egoísta) e do efeito sobre a soma dos tempos de viagem de todos os agentes do sistema, representando uma escolha altruísta. Investiga-se como diferentes combinações desses valores impactam nos níveis de congestionamento, de tempo de viagem e de comprimento de trajeto.

Essa investigação pode ajudar a entender como possíveis incentivos fornecidos aos agentes para influenciar suas decisões de escolha de trajeto impactariam na performance do sistema.

\section{Metodologia}

\section{Redes de transporte}

A representação de sistemas complexos em forma de grafos permite analisá-los com ferramentas da teoria de redes complexas. Segundo Barrat et al. (2008) um grafo é definido por um par de conjuntos $G=(V, E)$, onde $V$ é um conjunto de elementos denominado nós e $E$ é um conjunto de pares de elementos de $V$ chamados arestas. Se os pares do conjunto $E$ forem não ordenados, possuímos um grafo não direcionado. Caso contrário, o grafo é direcionado. A estrutura de um grafo pode ser representada matematicamente por uma matriz $A_{i j}$ chamada matriz de adjacência, cujas linhas e colunas $i$ e $j$ representam os nós e cujos elementos $a_{i j}$ são não-nulos se $i$ e $j$ são vizinhos. É possível atribuir a uma aresta um valor que indica uma característica da relação entre os nós. Em uma rede de transporte, uma representação comum trata nós como interseções e arestas como segmentos de ruas ou avenidas. Tempo de cruzamento, comprimento ou volume de fluxo são os valores mais comuns atribuídos às arestas em redes de transporte. A partir das arestas existentes entre os nós, é possível o surgimento de caminhos entre dois nós não adjacentes. O menor caminho entre dois nós é aquele cuja soma dos valores das arestas percorridas é o menor possível. Em redes de transporte, é comum que se utilize a soma dos tempos de cruzamento como função a ser minimizada, já que a minimização dos tempos de viagem é uma boa aproximação para o comportamento de motoristas na vida real.

O estudo de redes viárias com o uso de redes complexas pode enfatizar diferentes aspectos pertinentes do sistema de transporte. A robustez de uma rede em relação a falhas em cascata ou processos disruptivos foi abordada por exemplo em Perez e Pereira (2021), que avaliaram o impacto de falhas de semáforos, Jia et al. (2020) que estudaram falhas em cascata geradas por congestionamento, ou Casali e Heinimann (2020) que avaliaram a resiliência da malha rodoviária de Zurique usando conceitos emprestados da teoria de redes complexas. Estudos morfológicos acerca de redes viárias foram conduzidos por Boeing (2019) que investigou a distribuição angular entre as interseções de diversas redes de transporte, Ma et al. (2020) analisaram a presença de autossimilaridade representada por fractais na estrutura de redes de transporte chinesa. Serra e Hillier (2019) analisaram possíveis correlações métricas e angulares entre os elementos de redes de transporte. A teoria de redes complexas também já foi aplicada para otimizar, melhorar ou planejar mudanças nos sistemas de transporte, como Ding et al. (2019) que constrói um modelo de otimização de redes de transporte multimodal ou Melo Neto, Santos, Carvalho, Nascimento e Silva (2020) que investigaram a viabilidade de implementação de semáforos em cruzamentos específicos.

\section{Modelo de mobilidade}

Um volume $N$ de agentes será inserido no sistema com origem e destinos selecionados aleatoriamente. A cada vez que uma fração de $N$, chamada de $\gamma$ for gerada, os tempos de viagem dos agentes são atualizados em todas as arestas de agentes 
seleciona seus trajetos. Os agentes devem selecionar aquele trajeto da origem até o destino que minimize o custo da viagem. A cada agente gerado, uma unidade de fluxo é adicionada a cada aresta do caminho selecionado pelo agente. O impacto da mobilidade sobre o tempo de viagem de todos os agentes é dado pela função $S$, expressa na equação (1).

$$
S=\sum_{e}^{E} x_{e} t_{e}
$$

em que $x_{e}$ e $t_{e}$ são o volume de fluxo e o tempo de viagem na aresta $e$ pertencente ao conjunto de arestas da rede $E$.

A seleção de trajeto se dá por meio de uma função custo $C$ que o agente associa à cada aresta, ao qual o agente quer minimizar durante seu percurso. Essa função é dada na equação (2).

$$
C=(1-\delta) t_{i}(x)+\delta \frac{\partial S}{\partial x_{i}}
$$

em que $t_{i}$ é o tempo de viagem da aresta $i$ em função do fluxo $x_{i} \frac{\partial s}{\partial x_{i}}$ é o aumento marginal dos tempos de viagem de todos os usuários do sistema quando uma unidade de fluxo é adicionada e $\delta \leq 1$ é o parâmetro que controla o peso de cada componente do custo.

O tempo de viagem em função do fluxo de veículos é dado pela função BPR, apresentada na equação (3).

$$
t_{i}=t_{0 i}\left(1+\alpha\left(\frac{x_{i}}{c_{i}}\right)^{\beta}\right)
$$

em que $t_{0 i i}$ é o tempo ideal de trajeto considerando ausência de congestionamento e com o usuário utilizando a máxima velocidade permitida, $c_{i}$ é a capacidade de fluxo da via, $\alpha$ e $\beta$ são parâmetros cujos valores são iguais a 0.15 e 4 , respectivamente, com base na literatura. A função BPR foi aplicada em diversos trabalhos na literatura envolvendo engenharia de tráfego e redes de transporte. Para uma discussão mais aprofundada sobre as aplicações e características da função BPR, verificar Manzo (2013) ou Das e Rama Chilukuri (2020).

\section{Coleta de dados: análise da rede de transporte}

Segundo Boeing (2020), o OpenStreetMap é um mapa construído a partir da colaboração de usuários ao redor de todo o mundo. O OpenStreetMap é uma base que contém informações geográficas acerca de milhares de cidades, que vão desde a largura e o comprimento de ruas até a localização de comércios, hospitais, casas, árvores, etc. O OpenStreetMap não permite, no entanto, a conversão imediata dos dados em uma estrutura de grafo, tal que possa ser prontamente analisada pelas ferramentas da teoria de redes complexas. Para isto, foi criada a biblioteca OSMNx para a linguagem de programação Python. O OSMNx traz apenas funcionalidades básicas para a análise e manipulação de redes complexas, sendo necessário portanto o uso de uma ferramenta adicional para a simulação de seleção de trajetos, chamada Networkx. A estrutura de grafo fornecida pelo OSMNx é totalmente compatível com a biblioteca Networkx.

O OpenStreetMap não fornece estimativas acerca da capacidade de fluxo de cada segmento de rua, mas fornece informações sobre o tipo de via, o número de pistas e a largura do segmento. Com estas informações, é possível estimar a capacidade de fluxo com base no trabalho de Zilske et al. (2011). As capacidades de fluxo de cada tipo de via, bem como suas funções (em termos de engenharia de tráfego) são mostradas na Tabela 1. 
Tabela 1: Classificação e capacidade de estradas por tipo de via: OSM.

\begin{tabular}{|l|l|l|}
\hline Tipo de via & Definição do tipo de via (OpenStreetMap) & $\begin{array}{l}\text { Capacidade de fluxo } \\
\text { da via (veículos por } \\
\text { hora por pista) }\end{array}$ \\
\hline Auto-estrada & $\begin{array}{l}\text { Via de acesso restrito, normalmente com duas ou mais pistas e um acostamento de } \\
\text { emergência. }\end{array}$ & 2200 \\
\hline $\begin{array}{l}\text { Estrada } \\
\text { principal }\end{array}$ & As mais importantes estradas de uma cidade (depois das auto-estradas). & 2000 \\
\hline $\begin{array}{l}\text { Primária } \\
\text { Secundária }\end{array}$ & Fornecem mobilidade entre diferentes regiões dentro de centros urbanos. & 1000 \\
\hline Terciária & Ligam bairros pequenos dentro das regiões. & 1000 \\
\hline $\begin{array}{l}\text { Não- } \\
\text { classificada }\end{array}$ & Fornecem mobilidade dentro dos bairros. & 900 \\
\hline Residencial & Vias que ligam regiões residenciais ao resto da rede de transporte & 800 \\
\hline
\end{tabular}

Fonte: Zilske et al. (2011).

A porção central da rede de São Paulo pode ser observada na Figura 2. Para obtê-la no OSMNx, é preciso filtrar as vias baixadas tal que só os tipos que constam na Tabela 1 estejam presentes. Além disso, é preciso que só os elementos com o atributo "drive" sejam baixados, já que ciclovias e faixas de pedestre não terão influência para a simulação de fluxo de veículos. A porção central da rede de São Paulo abrange 2000 metros de distância radial a partir do ponto central da cidade.

Figura 1: Rede de transporte de São Paulo.

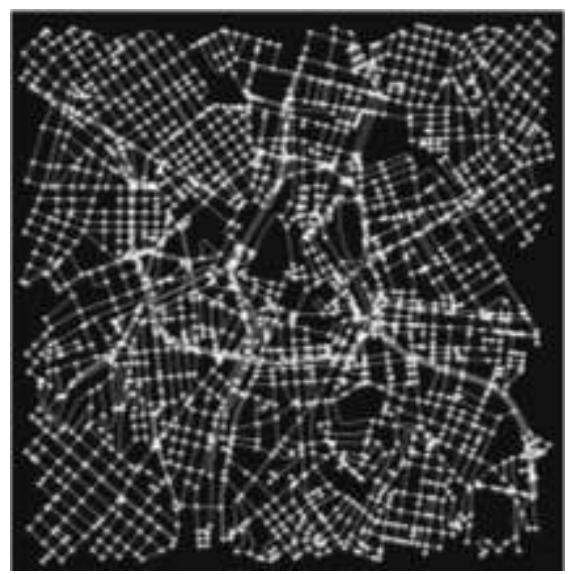

Fonte: Dos autores.

\section{Medidas de desempenho e planejamento dos experimentos}

Para entender como as diferentes estratégias impactam no desempenho da rede de transporte, foram analisadas as propriedades citadas na Tabela 2 . 
Tabela 2: Medidas de desempenho da simulação de mobilidade.

\begin{tabular}{|l|l|l|}
\hline Medida & Fórmula & Descrição \\
\hline Delay & $\sum_{i}^{E}\left(t_{i}-t_{0 i}\right)$ & $\begin{array}{l}\text { Soma das diferenças entre os tempos de viagem considerando } \\
\text { congestionamento e os tempos de viagem originais }\end{array}$ \\
\hline Tempo de Sistema & $\sum_{i}^{E} x_{i} t_{i}$ & Soma do tempo de viagem experimentado por todos os usuários no sistema. \\
\hline $\begin{array}{l}\text { Variação de comprimento } \\
\text { de trajeto }\end{array}$ & $\mu\left(\frac{l(p, \delta)}{l(p, 0)}\right)$ & $\begin{array}{l}\text { Média da razão entre os comprimentos de trajeto e a distância euclidiana entre } \\
\text { origem e destino para } \delta>0 \text { e } \delta=0 . \text { Como a distância euclidiana é a menor } \\
\text { distância entre dois pontos, ela é considerada a distância ideal. }\end{array}$ \\
\hline $\begin{array}{l}\text { Variação da duração de } \\
\text { trajeto }\end{array}$ & $\mu\left(\frac{t(p, \delta)}{t(p, 0)}\right)$ & $\begin{array}{l}\text { Média da razão entre o tempo efetivo e o tempo ideal de trajeto para } \delta>0 \text { e } \delta \\
=0\end{array}$ \\
\hline
\end{tabular}

Fonte: Dos autores.

$\mathrm{O}$ valor de $\delta$ varia de 0 até 1 em incrementos de 0.05 , para um mesmo conjunto de origens e destinos selecionados aleatoriamente, o que equivale a 20 pontos. Após isso, um novo conjunto de origens e destinos é selecionado e o processo recomeça. Este ciclo foi replicado dez vezes. O número de agentes selecionado foi 150000 , enquanto o valor de $\gamma$ foi fixado em 0.1 .

\section{Resultados e Discussão}

Nesta seção são apresentados e discutidos os resultados obtidos na simulação. A Figura 2 apresenta os resultados para o tempo de sistema e para o delay. 
Figura 2 - Tempo de sistema e delay para diferentes valores de $\delta$.
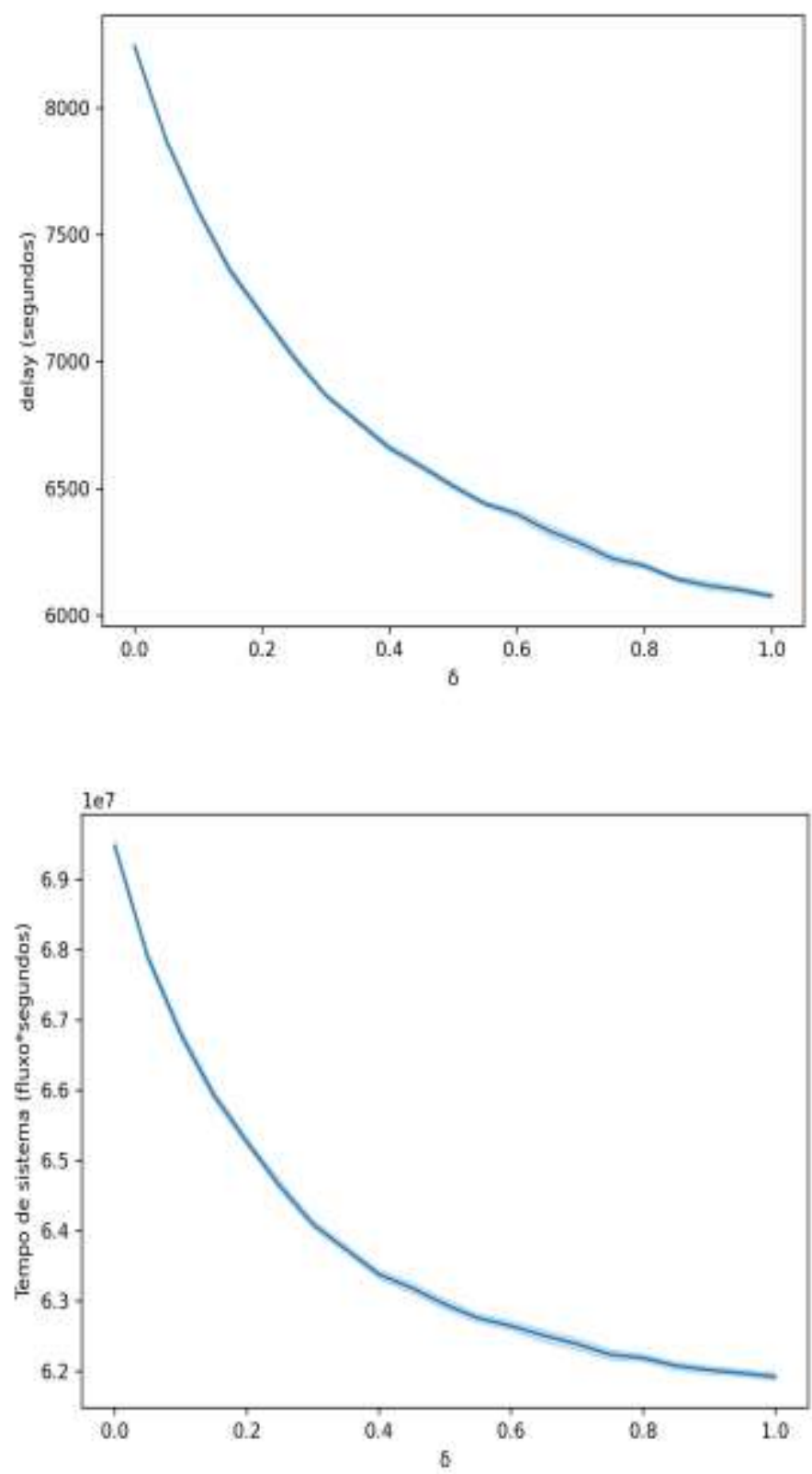

Fonte: Dos autores.

Percebe-se que a adoção de trajetos que priorizam a minimização da sobrecarga sobre o sistema reduz o delay em até 30 minutos, considerando um volume de fluxo de 150000 trajetos realizados, enquanto o tempo de sistema é reduzido em aproximadamente $20 \%$ do valor obtido para trajetos puramente egoístas.

A redução sobre variáveis do sistema ajuda a perceber os potenciais benefícios de se incorporar nos processos de decisão de trajeto considerações sobre a carga imposta ao sistema de transporte. Mas, para a grande maioria dos usuários, a decisão sobre qual trajeto selecionar é individual e auto-centrada, portanto, deve haver benefícios perceptíveis a nível individual a fim de tornar a adoção de trajetos altruístas uma estratégia mais eficiente. Para observar como, para o usuário individual, a adoção de trajetos altruístas impacta nas características de suas viagens, foram analisadas as variações de comprimento e de tempo de trajeto. Os resultados para essas variáveis estão presentes na Figura 3. 
Figura 3 - Variações de comprimento de trajeto e de tempo em trajeto em relação a trajetos puramente egoístas.

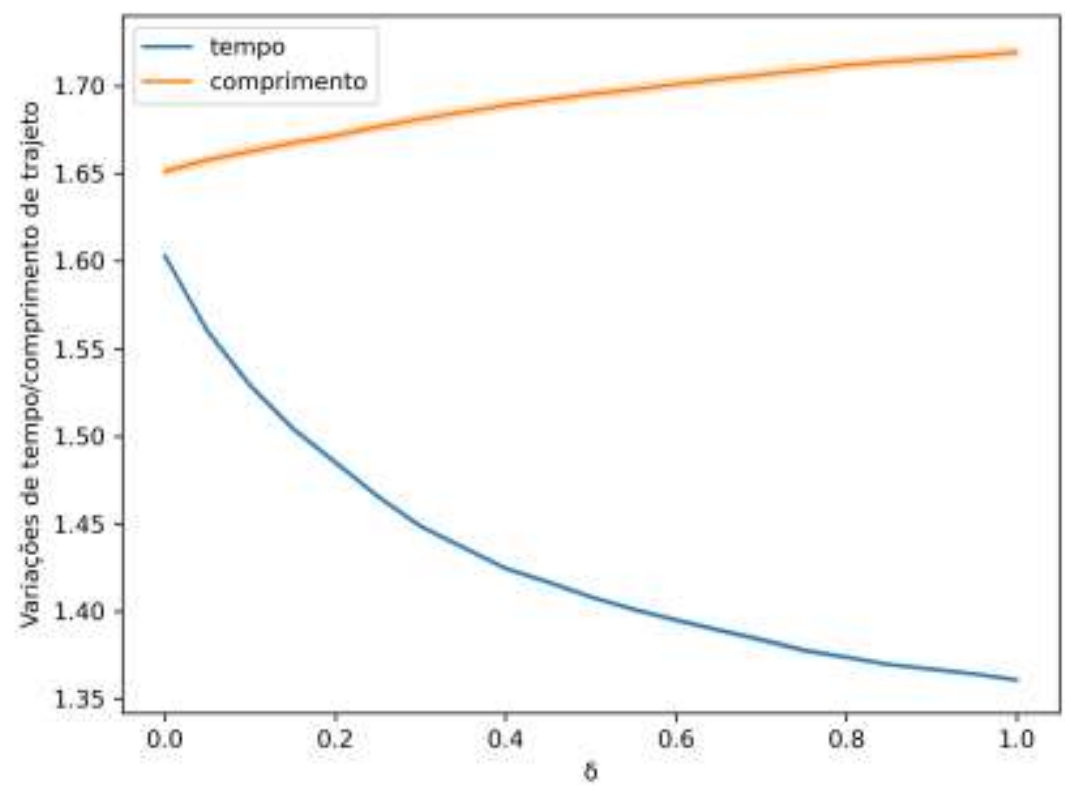

Fonte: Dos autores.

É possível perceber que a razão entre comprimento de trajeto e distância euclidiana aumenta em aproximadamente 5\% com a adoção de trajetos altruístas, enquanto a razão entre o tempo de viagem efetivo e o tempo de viagem ideal cai de, aproximadamente, 1.6 para 1.35. Estes resultados indicam que, embora tenha havido uma perda de eficiência de trajeto no quesito distância, houve um ganho de eficiência muito mais significante no quesito tempo. Este ganho de eficiência pode ser explorado para construir políticas de incentivo ao uso de trajetos mais eficientes de uma perspectiva altruísta.

Para melhor demonstrar as diferenças nos dois meios de trajeto, construiu-se a partir do grafo da rede de transporte um grafo auxiliar onde dois pontos da rede de transporte estão ligados entre si se houver um caminho entre eles e este grafo será chamado de $H$. Como peso para as arestas de $H$ foi selecionado o tempo de trajeto. Após a simulação, o grafo $H$ teve removidas todas as aresttas cujo razão entre o tempo de trajeto original e o tempo de trajeto efetivo foi maior ou igual a 0.6. Isto permitiu que só aqueles trajetos que tiveram maior redução de velocidade permanecessem.

Por fim, após a remoção dessas arestas, foi extraído o subgrafo do maior componente fortemente conectado, isto é, o maior subgrafo onde é possível encontrar um trajeto entre dois pontos. Para $\delta=0 H$ possui 22357 arestas e seu maior componente fortemente conectado possui 19158 arestas, enquanto para $\delta=1 H$ possui 3420 arestas e seu maior componente fortemente conectado é 931. Uma ilustração dos dois subgrafos obtidos para cada valor de $\delta$ é apresentada na Figura 4. 
Figura 4: Maior componente fortemente conectado do grafo $H$ para a) $\delta=0$ e para b) $\delta=1$

a) $\delta=0$.

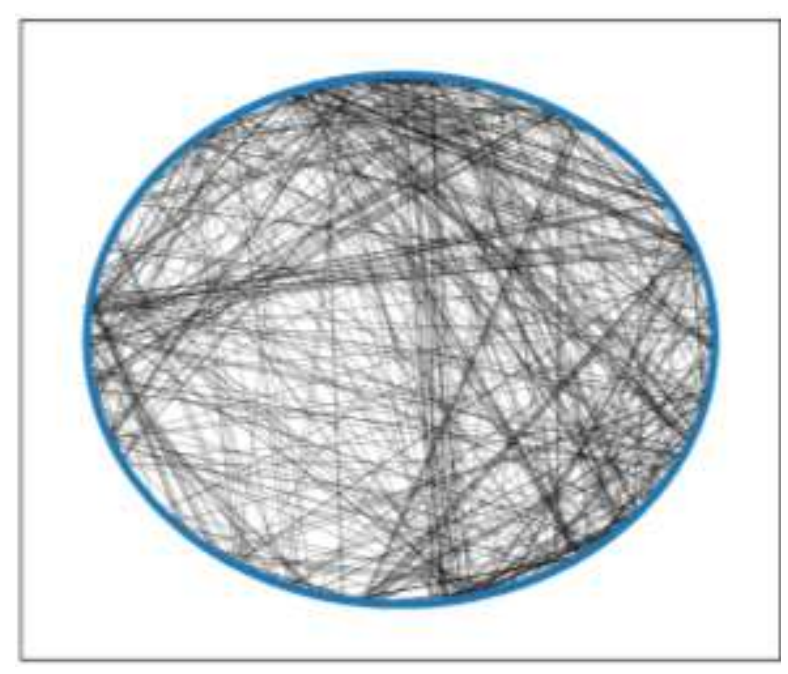

b) $\delta=1$

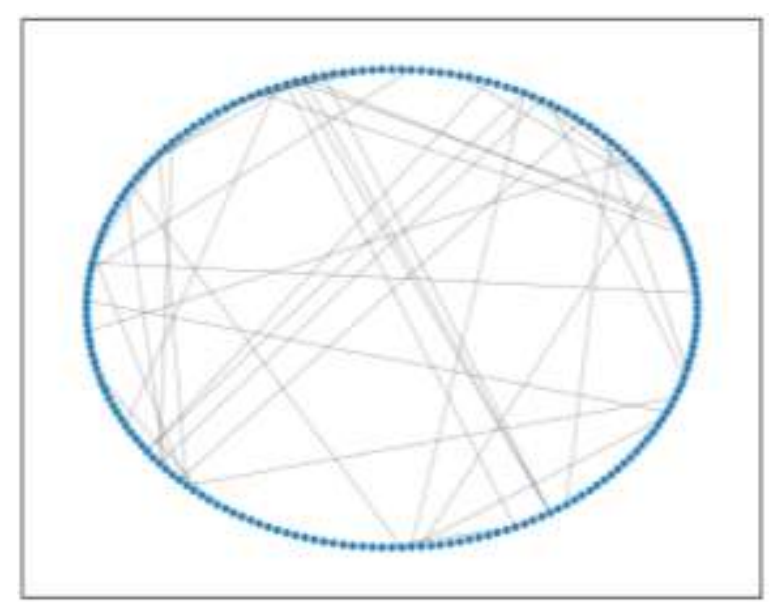

Fonte: Dos autores.

\section{Conclusão}

Há duas informações importantes obtidas pela análise do maior componente fortemente conectado de $H$. A primeira é que $H$ é muito maior para $\delta=0$ do que para $\delta=1$, indicando que há muito mais trajetos congestionados cujo tempo de viagem se modificou mais intensamente. A segunda é que os trajetos congestionados formam agrupamentos mais coesos para $\delta=0$, de modo que o componente fortemente conectado de $H$ para $\delta=0$ tem quase tantas arestas quanto $H$. Estes agrupamentos podem ser encarados como ilhas de congestionamento que vão se espalhando pelo sistema conforme a simulação de mobilidade prossegue. Já para $\delta=1$ é possível notar uma redução brusca de $H$ e do maior componente fortemente conectado de $H$, indicando que não só há menos trajetos cujo tempo de viagem se reduziu significativamente como também que os trajetos congestionados são mais pontuais e localizados. A redução dos níveis de delay causados por congestionamento se reflete também no uso de combustíveis como a gasolina e na melhoria da saúde dos cidadãos. A redução 
de tempos de viagem em redes de transporte urbano pode ter vários benefícios para a sociedade como um todo, como a redução da emissão de poluentes e de acidentes ou na redução dos níveis de stress dos cidadãos.

\section{Referências}

Auld, J., Verbas, O., \& Stinson, M. (2019). Agent-based dynamic traffic assignment with information mixing. Procedia Computer Science, 151, 864-869.

Barrat, A., Barthelemy, M., \& Vespignani, A. (2008). Dynamical processes on complex networks. Cambridge university press.

Barthelemy, J., \& Carletti, T. (2017). A dynamic behavioural traffic assignment model with strategic agents. Transportation Research Part C: Emerging Technologies, 85, 23-46. Ben-Akiva, M. E., Gao, S., Wei, Z., \& Wen, Y. (2012). A dynamic traffic assignment model for highly congested urban networks. Transportation research part C: emerging technologies, 24, 62-82.

Ben-Akiva, M. E., Gao, S., Wei, Z., \& Wen, Y. (2012). A dynamic traffic assignment model for highly congested urban networks. Transportation research part C: emerging technologies, 24, 62-82. Ben-Akiva, M. E., Gao, S., Wei, Z., \& Wen, Y. (2012). A dynamic traffic assignment model for highly congested urban networks. Transportation research part $C$ : emerging technologies, 24, 62-82.

Boeing, G. (2019). Urban spatial order: Street network orientation, configuration, and entropy. Applied Network Science, 4(1), 1-19.

Boeing, G. (2020). A multi-scale analysis of 27,000 urban street networks: Every US city, town, urbanized area, and Zillow neighborhood. Environment and Planning B: Urban Analytics and City Science, 47(4), 590-608.

Casali, Y., \& Heinimann, H. R. (2020). Robustness response of the Zurich road network under different disruption processes. Computers, Environment and Urban Systems, 81, 101460.

Crucitti, P., Latora, V., \& Porta, S. (2006). Centrality measures in spatial networks of urban streets. Physical Review E, 73(3), 036125. Eikenbroek, O. A., Still, G. J., \& van Berkum, E. C. (2021). Improving the performance of a traffic system by fair rerouting of travelers. European Journal of Operational Research.

Das, A. K., \& Rama Chilukuri, B. (2020). Link Cost Function and Link Capacity for Mixed Traffic Networks. Transportation Research Record, 2674(9), 3850 .

Ding, R., Yin, J., Dai, P., Jiao, L., Li, R., Li, T., \& Wu, J. (2019). Optimal Topology of Multilayer Urban Traffic Networks. Complexity, 2019.

Eikenbroek, O. A Still, G. J., \& van Berkum, E. C. (2021). Improving the performance of a traffic system by fair rerouting of travelers. European Journal of Operational Research.Eikenbroek, O. A., Still, G. J., \& van Berkum, E. C. (2021). Improving the performance of a traffic system by fair rerouting of travelers. European Journal of Operational Research.Gao, J., Barzel, B., \& Barabási, A. L. (2016). Universal resilience patterns in complex networks. Nature, 530(7590), 307-312.

Gao, J., Barzel, B., \& Barabási, A. L. (2016). Universal resilience patterns in complex networks. Nature, 530(7590), 307-312. Gao, J., Barzel, B., \& Barabási, A. L. (2016). Universal resilience patterns in complex networks. Nature, 530(7590), 307-312.

Helbing, D. (Ed.). (2012). Social self-organization: Agent-based simulations and experiments to study emergent social behavior. Springer.

Jia, H., Li, F., Yang, L., Luo, Q., \& Li, Y. (2020). Dynamic Cascading Failure Analysis in Congested Urban Road Networks With Self-Organization. IEEE Access, 8, 17916-17925.Ladyman, J., Lambert, J., \& Wiesner, K. (2013). What is a complex system?. European Journal for Philosophy of Science, 3(1), 3367.

Ladyman, J., Lambert, J., \& Wiesner, K. (2013). What is a complex system?. European Journal for Philosophy of Science, 3(1), 33-67. Ladyman, J., Lambert, J., \& Wiesner, K. (2013). What is a complex system?. European Journal for Philosophy of Science, 3(1), 33-67.

Levy, N., Klein, I., \& Ben-Elia, E. (2018). Emergence of cooperation and a fair system optimum in road networks: A game-theoretic and agent-based modelling approach. Research in Transportation Economics, 68, 46-55.

Liu, W., \& Song, Z. (2020). Review of studies on the resilience of urban critical infrastructure networks. Reliability Engineering \& System Safety, 193, 106617.

Ma, D., Guo, R., Zheng, Y., Zhao, Z., He, F., \& Zhu, W. (2020). Understanding Chinese urban form: the universal fractal pattern of street networks over 298 cities. ISPRS International Journal of Geo-Information, 9(4), 192.

Macal, C. M., \& North, M. J. (2005). Validation of an agent-based model of deregulated electric power markets. In Proc. North American Computational Social and Organization Science (NAACSOS) 2005 Conference, South.

Manzo, S., Nielsen, O. A., \& Prato, C. G. (2013). Investigating uncertainty in BPR formula parameters: a case study.

Melo Neto, O. de M., Santos, B. L. de F., Carvalho, F. do S. de S., Nascimento, A. M. V. do, \& Silva, G. C. B. da. (2020). Urban planning: traffic feasibility near rotating through a software to improve flow of vehicles and pedestrians. Research, Society and Development, 9(7), e13973808. https://doi.org/10.33448/rsd-v9i7.3808

Moreira, L. de A., Santos, S. F. dos, Oliveira Neto, R. de, \& Silva Junior, L. A. (2019). Bibliographic review of the mode of road transportation in Brazil. Research, Society and Development, 8(3), e2283728. https://doi.org/10.33448/rsd-v8i3.728

Perez, Y., \& Pereira, F. H. (2021). Simulation of traffic light disruptions in street networks. Physica A: Statistical Mechanics and its Applications, 582 , 126225.

Portugali, J. (2016). What makes cities complex?. In Complexity, cognition, urban planning and design (pp. 3-19). Springer, Cham. 
Research, Society and Development, v. 10, n. 14, e546101422514, 2021

(CC BY 4.0) | ISSN 2525-3409 | DOI: http://dx.doi.org/10.33448/rsd-v10i14.22514

Rambha, T., Boyles, S. D., Unnikrishnan, A., \& Stone, P. (2018). Marginal cost pricing for system optimal traffic assignment with recourse under supply-side uncertainty. Transportation Research Part B: Methodological, 110, 104-121.

Salman, S., \& Alaswad, S. (2018). Alleviating road network congestion: Traffic pattern optimization using Markov chain traffic assignment. Computers \& Operations Research, 99, 191-205.

Serra, M., \& Hillier, B. (2019). Angular and metric distance in road network analysis: A nationwide correlation study. Computers, Environment and Urban Systems, 74, 194-207.

Sharifi, A. (2019). Resilient urban forms: A macro-scale analysis. Cities, 85, 1-14.Sharifi, A. (2019). Resilient urban forms: A macro-scale analysis. Cities, 85, 1-14. Zilske, M., Neumann, A., \& Nagel, K. (2015). OpenStreetMap for traffic simulation. Technische Universität Berlin.

Zilske, M., Neumann, A., \& Nagel, K. (2015). OpenStreetMap for traffic simulation. Technische Universität Berlin.Zilske, M., Neumann, A., \& Nagel, K. (2015). OpenStreetMap for traffic simulation. Technische Universität Berlin. 